\title{
Numerical Modeling of the Flow in a Vaneless Diffuser of Centrifugal Compressor Stage
}

\author{
Mykola Kalinkevych and Oleg Shcherbakov \\ Technical Thermophysics Department, Sumy State University, 2 Rymskogo-Korsakova Street, Sumy 40007, Ukraine \\ Correspondence should be addressed to Oleg Shcherbakov; ang81@yandex.ru
}

Received 1 August 2013; Accepted 19 August 2013

Academic Editors: W.-H. Chen and G. Juncu

Copyright ( 2013 M. Kalinkevych and O. Shcherbakov. This is an open access article distributed under the Creative Commons Attribution License, which permits unrestricted use, distribution, and reproduction in any medium, provided the original work is properly cited.

\begin{abstract}
This paper presents the results of numerical investigation of the flow in a vaneless diffuser of centrifugal compressor stage. Simulations were performed using both a commercial CFD package ANSYS CFX and the own-designed computer program. Steady conditions involving SST turbulence model were used for the calculations using CFX. To consider the interaction between impeller and diffuser, not just a diffuser but the whole stage was calculated. The own-designed methodology is based on solving of conservation equations with assumptions that flow in a diffuser is steady state and axisymmetric. The flow area is divided into the flow core and boundary layers. Results of calculation were compared with experimental data.
\end{abstract}

\section{Introduction}

The behavior of vaneless diffusers of centrifugal compressors has been widely studied theoretically, experimentally, and numerically. Theoretical analyses have been carried out by Jansen [1], Senoo and Kinoshita [2], Tsujimoto et al. [3], Ljevar et al. [4], and so forth. Experimental measurements have been performed by Nuzhdin [5], Abdelhamid [6], Kinoshita and Senoo [7], Jaatinen-Varri et al. [8], and so forth. It was shown that stages with vaneless diffusers have a wide operating range and high polytropic efficiency at high flow rates; however, at low flow rates their efficiency extremely decreases because of flow separation and rotating stall inception. The last one results in dramatically loss of compressor performance and instability and even can cause damage of the machine. Numerical simulations of vaneless diffusers have been performed by Gao et al. [9], Khalfallah and Ghenaiet [10], Izmailov et al. [11], Tamaki [12], and others. Obtained numerical results show good agreement with the experimental ones, but all the authors have faced the problem of rotor/stator interaction.

This paper presents the results of both experimental and numerical investigation of a centrifugal compressor vaneless diffuser. Simulations were performed using both commercial CFD package Ansys CFX and the own-designed computer program. Investigation has been performed to predict the overall performance of a diffuser and to obtain flow patterns. Finally, the flow structure was analyzed to identify and quantify the sources of losses.

\section{Experimental Procedure}

First of all an experimental investigation of the flow has been performed. The test vaneless diffuser is an element of the Sumy Frunze NPO gas compressor end stage. Cross-section of the diffuser is shown in Figure 1(a). Diffuser width ratio is $b_{3} / b_{2}=1.07$.

The air entered the test stage in axial direction through the suction pipe with a filter and an orifice plate. The inlet total and static pressures and temperatures were measured. The operation point was set by a throttle valve installed at the discharge pipe. The mass flow rate was measured with the orifice plate installed at the suction pipe. Also ambient pressure, temperature, and humidity were measured. To obtain the flow fields inside of the diffuser and to estimate its overall performance the following parameters were measured. Total pressures and flow angles were measured with the three-hole probe at eight radial positions downstream of the impeller. The probe was traversed across the diffuser at five points at each of the diameter. The probe was calibrated over a broad 


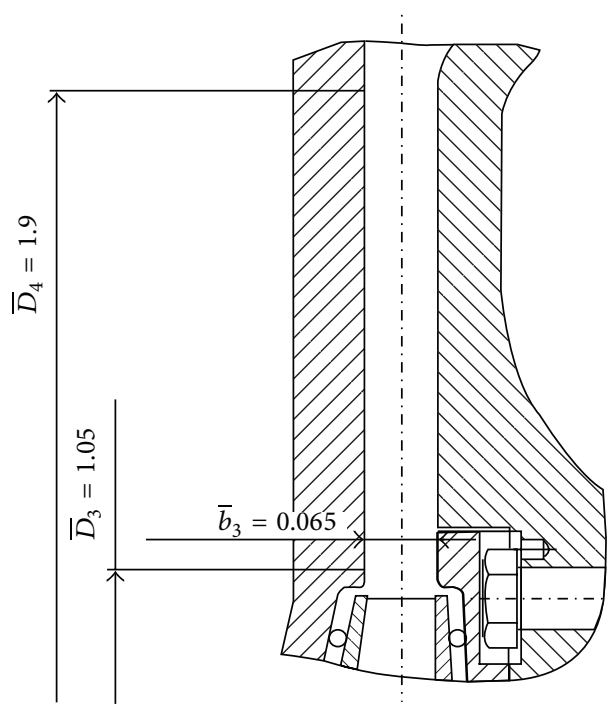

(a)

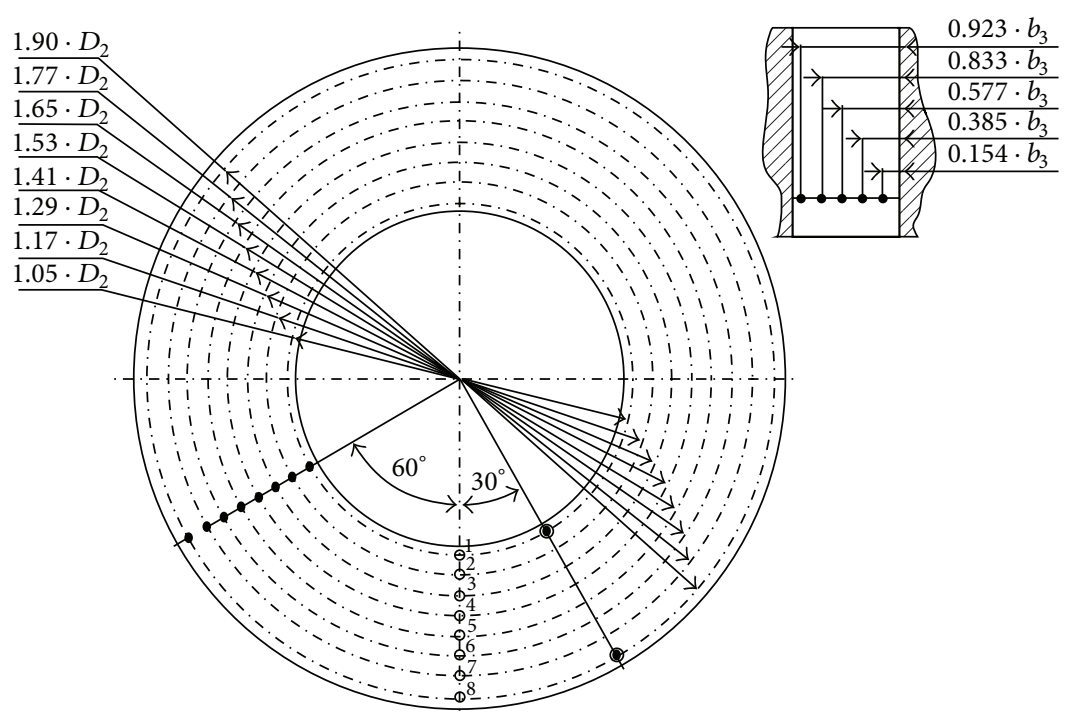

(b)

Figure 1: Cross-section of the test diffuser (a) and the locations of the probe traverses, static pressure taps, and thermocouples (b), and $\bar{b}=b / D_{2}, \bar{D}=D / D_{2}$.

Mach number range. Static pressures were also measured at the same diameters through the holes in the diffuser shroud wall. Total temperatures were measured with thermocouples at the diffuser inlet and outlet. To eliminate an influence of the scroll tongue the checkout tests were performed before the main ones. Final location of the static pressure taps, probe traverses, and thermocouples are shown in Figure 1(b). Tests were performed at the design speed. The experimental data was gathered until surge becomes evident. The onset of surge was determined by the level of pressure pulsations.

The overall performance of the diffuser was evaluated using the static pressure recovery and the total pressure loss coefficients. The static pressure recovery coefficient was defined as

$$
C_{p}=\frac{p_{4}-p_{3}}{p_{3}^{*}-p_{3}}
$$

and the total pressure loss coefficient was defined as

$$
\zeta=\frac{p_{3}^{*}-p_{4}^{*}}{p_{3}^{*}-p_{3}}
$$

where $p_{3}^{*}$ and $p_{3}$ are the averaged total and static pressures at the diffuser inlet, whereas $p_{4}^{*}$ and $p_{4}$ are the averaged total and static pressures at the diffuser outlet.

The detailed description of the test system and procedure was given by Kalinkevych and Shcherbakov [13].

\section{Flow Simulation Procedure}

Numerical investigation was performed in addition to experimental investigation for better understanding of the flow structure.
Flow in a diffuser substantially depends on the flow behavior at the impeller outlet. Therefore, to take into account an influence of the impeller, the two-element stage (impeller and vaneless diffuser) was modeled. A steady state model was used for all calculations. The stage and frozen rotor interfaces were used for the calculations involving SST turbulence model. The stage interface was used to calculate the overall diffuser performance and to predict the flow structure at the diffuser, whereas the frozen rotor simulation was used for prediction of the flow patterns at the impeller.

The computational domain contained two different meshes. The impeller mesh is a structured hexahedral mesh, generated using ANSYS TurboGrid. An H/J/C/L-Grid including an $\mathrm{O}-\mathrm{Grid}$ topologies were used. The mesh was refined to better resolve the flow in the vicinity of the leading and trailing edges of blades. A refinement was also performed near the hub and shroud walls. To prevent a mesh dependent error the grid independence analysis has been performed. Five mesh sizes in the range of 200 to 600 thousand nodes have been generated for the impeller. As a result, the mesh with 430 thousand nodes has been selected for all simulations. Near wall nodes were positioned in the way to ensure the value of $y+$ not more than 2.5. The resulting mesh is shown in Figure 2.

The diffuser was meshed with an unstructured tetrahedral mesh with prism layers along the wall surfaces to resolve the near-wall boundary layer flow. Mesh generation was performed using ANSYS CFX-Mesh. On the analogy of the impeller five mesh sizes have been generated for the diffuser to perform the grid independence analysis. Consequently, the mesh with 1.1 million nodes has been selected. The final mesh is presented in the Figure 3.

Experimentally measured total pressure and total temperature were specified at the impeller inlet boundary and the 


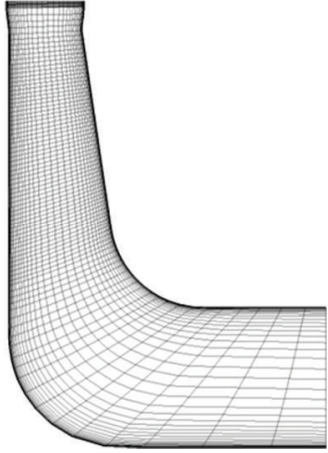

(a)

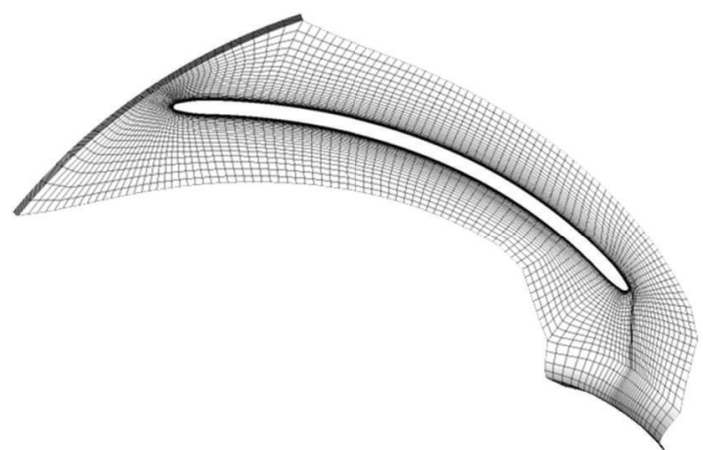

(b)

FIGURE 2: Impeller mesh: (a) meridional view; (b) midspan view.

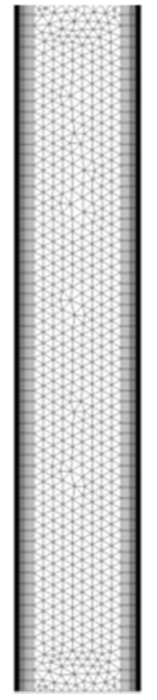

(a)

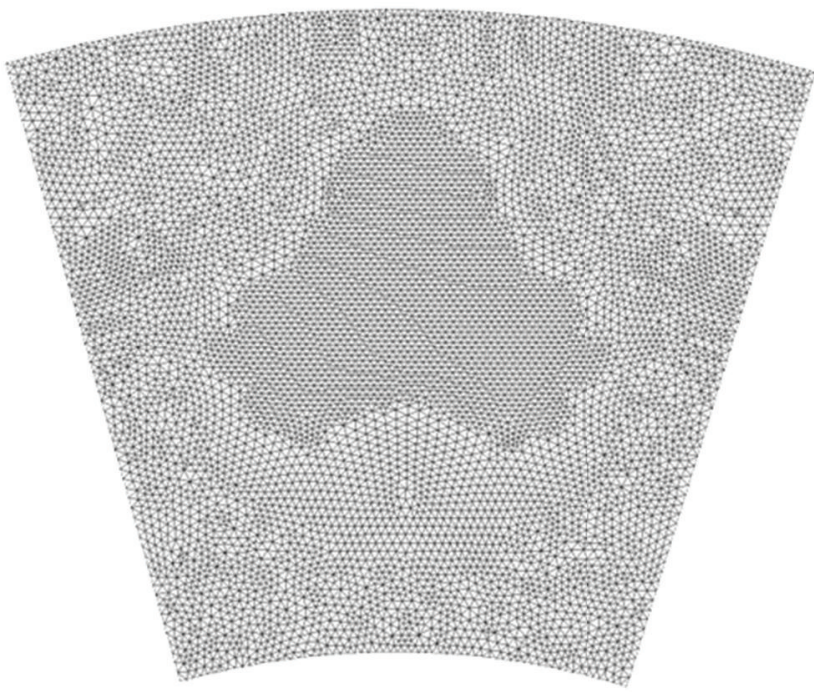

(b)

FIGURE 3: Diffuser mesh: (a) meridional view; (b) midspan view.

mass flow rate at the diffuser outlet. Nonslip and adiabatic conditions were imposed all over the solid walls. The periodic boundaries were specified for the lateral sides of impeller and diffuser domains.

Because surge and rotating stall are unsteady phenomena that cannot be modeled as a steady state problem, simulations were performed for the flow rates at which stall does not initiate.

\section{The Own-Designed Model}

A new vaneless diffuser calculation procedure based on the boundary-layer theory has been developed and presented by Kalinkevych et al. [14]. The developed method allows to calculate flow parameters and diffuser performance and to predict the boundary-layer separation. The flow was assumed to be steady state and circumferentially uniform, and inlet velocities were assumed to be invariable by the width of the diffuser. According to the boundary layer theory the flow area was divided into the flow core and boundary layers. The simplified model of the boundary layer was accepted for the mathematical description of the flow behavior. First of all, time-averaged boundary layer parameters were considered, while the turbulent pulsations were taken into account using additional stresses. Under the accepted model, a boundary layer was divided into two regions: the so called viscous sublayer near the walls in which the flow is laminar and the region of turbulent flow in which both molecular and molar friction forces exist. Velocity profiles described by Sherstyuk [15] were used for the calculation of the flow field. Viscous sublayer velocity profile is

$$
\begin{aligned}
v=2 \sqrt{\frac{\beta_{\text {sub }}}{\chi}} & \\
& \times \int_{0}^{z_{1}} \frac{1}{z_{1}^{4}}\left[\sqrt{1+z_{1}^{4}\left(1+\frac{A_{*}}{\mathrm{Re}_{*}} \sqrt{\frac{\beta_{\text {sub }}}{\chi}} z_{1}\right)}-1\right] d z_{1},
\end{aligned}
$$




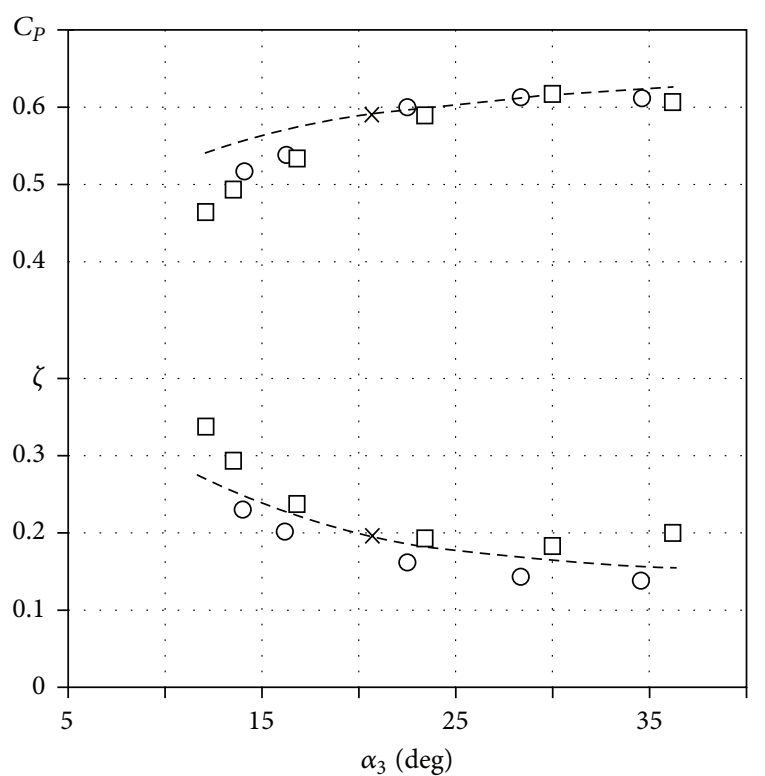

FIGURE 4: Diffuser performance: $\square \square \square$ measured data; ooo Ansys CFX data; - - the own-designed model data $(\times-$ the predicted separation point).

where $\beta=c_{*} \cdot z / v ; \beta_{\text {sub }}=c_{*} \cdot \delta_{\text {sub }} / v ; z_{1}=\sqrt{\chi / \beta_{\text {sub }}} \cdot \beta ; A_{* r}=$ $\left(\delta / \rho c_{* r}^{2}\right) \cdot(d p / d r) ; A_{* u}=0$.

According to Sherstyuk [15] at the edge of the viscous sublayer $\operatorname{Re}_{\text {sub }}=c \cdot \delta_{\text {sub }} / v=56$. The Reynolds number was calculated for the absolute flow velocity $c=\sqrt{c_{r}^{2}+c_{u}^{2}}$.

At $\eta<0.1$ the following velocity profile was used:

$$
\begin{aligned}
v=v_{\text {sub }}+\frac{1}{\chi} \int_{z_{2 \text { sub }}}^{z_{2}} & {\left[\sqrt{\frac{1}{z_{2}^{4}}+\frac{1}{z_{2}^{2}}\left[1+\frac{A_{*}}{\mathrm{Re}_{*}}\left(\frac{z_{2}}{2 \chi}+\beta_{\text {sub }}\right)\right]}\right.} \\
& \left.-\frac{1}{z_{2}^{2}}\right] d z_{2}
\end{aligned}
$$

where $z_{2}=2 \chi\left(\beta-\beta_{\mathrm{sub}} / 2\right)$.

Turbulent flow region velocity profile (at $0.1 \leq \eta<1$ ) is

$$
\frac{d v}{d \eta}=\frac{1}{\chi} \frac{\sqrt{(1-\eta)\left(1+\left(1+A_{*}\right) \eta\right)}}{\left(\eta-\eta_{\text {sub }}\right)\left(1-\left(\eta /\left(2-\eta_{\text {sub }}\right)\right)\right)} .
$$

Friction velocities $c_{r^{*}}, c_{u^{*}}$ were calculated using iterative method:

$$
C_{r}=\frac{1}{b} \int_{0}^{b} c_{r} d z ; \quad C_{u}=\frac{1}{b C_{r}} \int_{0}^{b} c_{u} c_{r} d z
$$

After that the displacement thickness and the momentum thickness could be calculated numerically:

$$
\delta_{r}^{*}=\int_{0}^{\delta}\left(1-\frac{c_{r}}{c_{r m}}\right) d z ; \quad \delta_{r}^{* *}=\int_{0}^{\delta} \frac{c_{r}}{c_{r m}}\left(1-\frac{c_{r}}{c_{r m}}\right) d z .
$$

The Buri shape factor was used as a separation criterion:

$$
\Gamma=\frac{\delta_{r}^{* *}}{\rho \cdot c_{r m}} \frac{d p}{d r}\left(\frac{\delta_{r}^{* *} \cdot c_{r m}}{\nu}\right)^{0.25} .
$$

Separation occurs if

$$
\Gamma<\Gamma_{0}=-0.06 .
$$

\section{Results}

The calculated and measured total pressure loss and static pressure recovery coefficients, presented as a function of the inlet flow angle in stationary frame $\alpha_{3}$, are shown in Figure 4 . The aerodynamic performance of the diffuser was estimated using mass flow averaged total pressure and area-averaged static pressure at the inlet and outlet of the diffuser.

The diffuser inlet flow angle was defined as

$$
\alpha_{3}=\operatorname{arctg}\left(\frac{C_{r 3}}{C_{u 3}}\right),
$$

where $C_{r 3}$ and $C_{u 3}$ are averaged radial and circumferential velocities at the diffuser inlet.

As the diagram shows there is a point with the minimum measured total pressure loss coefficient at $\alpha_{3}=30.0^{\circ}$, while the calculated ones monotonically decrease as the mass flow rate increases. The average discrepancy between the measured and predicted total pressure loss coefficients is $17.3 \%$ for Ansys CFX and 14.5\% for the own-designed methodology. The maximum discrepancy is $31.2 \%$ and $27.2 \%$ for Ansys CFX and for the own-designed methodology, respectively. Concerning the calculated static pressure recovery coefficients, they are in good agreement with the experimental results. The average discrepancy between the measured and predicted static pressure recovery coefficients is $2.3 \%$ for Ansys CFX and $4.7 \%$ for the own-designed methodology. The maximum discrepancy is 3.4\% and 7.8\% for Ansys CFX and for the owndesigned methodology, respectively.

An increase in total pressure loss at low flow angles may be caused by higher flow velocities at the impeller outlet, because of higher specific work at low flow rate, resulting in higher wall friction losses.

To identify other sources of losses, velocity diagrams were considered. The measured radial velocity diagrams are shown in Figure 5. As the diagram shows there is a flow separation zone at the diffuser hub at low flow rates. Flow separation results in decrease in the total pressure. As the mass flow rate decreases this zone becomes more prominent, which causes higher total pressure losses. In addition, flow separation reduces the effective flow passage. As a result the flow velocity at the shroud is accelerated, which also produces an increase in total pressure loss coefficient.

The measured flow diagrams agree quite well with the numerical results. Figure 6 shows the streamline patterns in the diffuser meridional plane at different inlet flow angles. At $\alpha_{3}=22.4^{\circ}$ a mild separation zone at the diffuser hub near the outlet was detected, which was considerably enlarged at $\alpha_{3}=16.8^{\circ}$. 


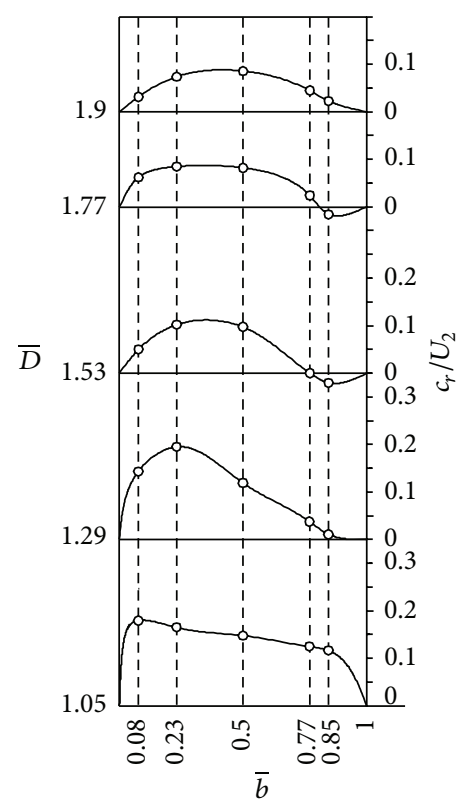

(a) $\alpha_{3}=16.8^{\circ}$

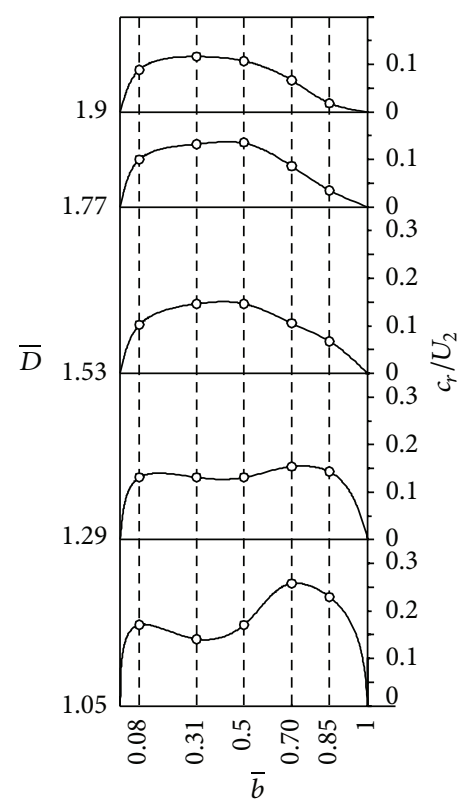

(b) $\alpha_{3}=22.4^{\circ}$

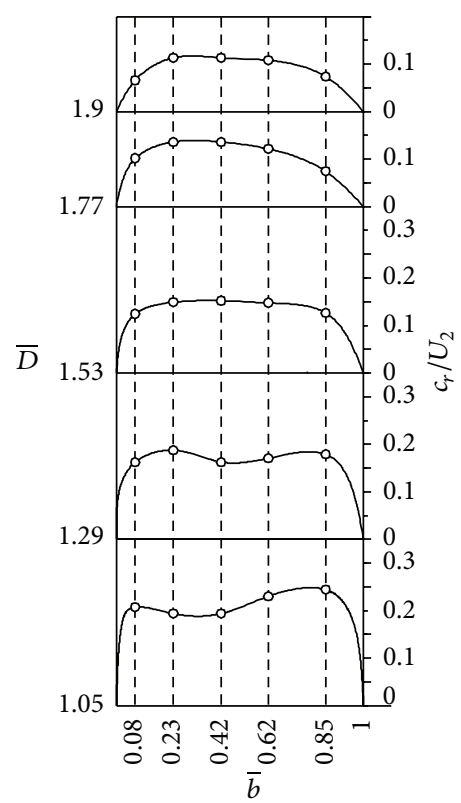

(c) $\alpha_{3}=30.0^{\circ}$

FIgURE 5: The measured dimensionless radial velocities.

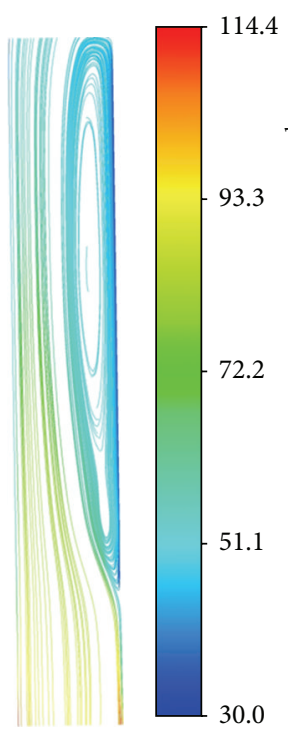

(a) $\alpha_{3}=16.8^{\circ}$

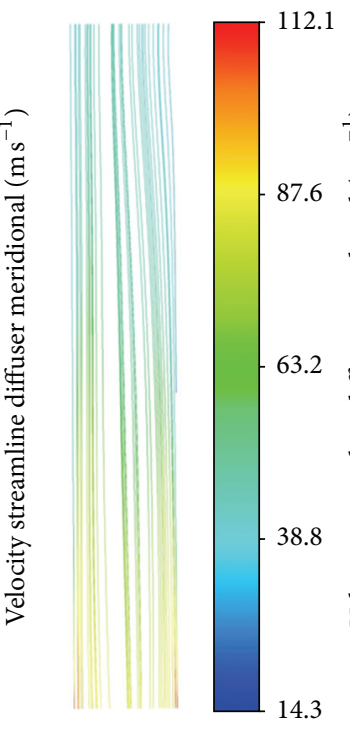

(b) $\alpha_{3}=22.4^{\circ}$

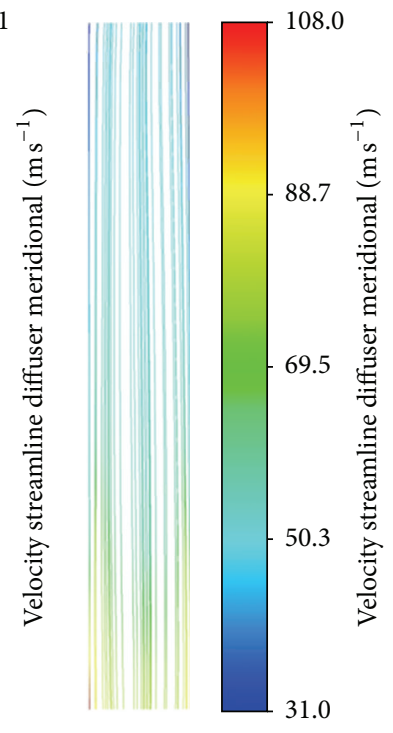

(c) $\alpha_{3}=30.0^{\circ}$

FIGURE 6: Diffuser streamlines in meridional plane.

The own-designed model has also predicted that at $\alpha_{3}=$ $21.1^{\circ}$ the separation initiates. So it can be concluded that separation point is predicted satisfactory. The location where separation first appears is shown in Figure 4 as " $x$ ".

Figure 7 shows the change in measured and calculated average total pressure through the diffuser, identifying the regions of total pressure losses. As the experimental results show, there is a significant decrease in total pressure at the diffuser inlet. This is due to intensive jet-wake mixing downstream of the impeller and because of higher velocities, resulting in higher friction losses. Dean and Senoo [16] first proposed a nonuniform jet-wake model for vaneless diffuser inlet flow and suggested that nonuniformity can affect flow behavior. Their theory predicted a significant, reversible work transfer between the jet and wake flows, leaving the impeller and entering the vaneless diffuser. As the flow passes through the diffuser, flow becomes homogenous, so friction and separation losses dominate. As for the calculated results, steady state simulations do not allow model jet-wake mixing, and that is why calculated total pressures are higher than the 


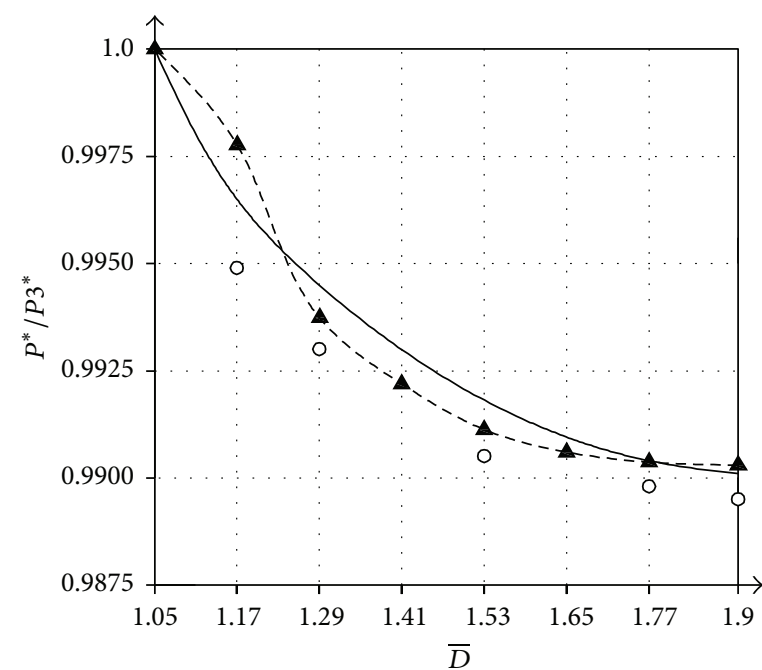

(a) $\alpha_{3}=16.8^{\circ}$

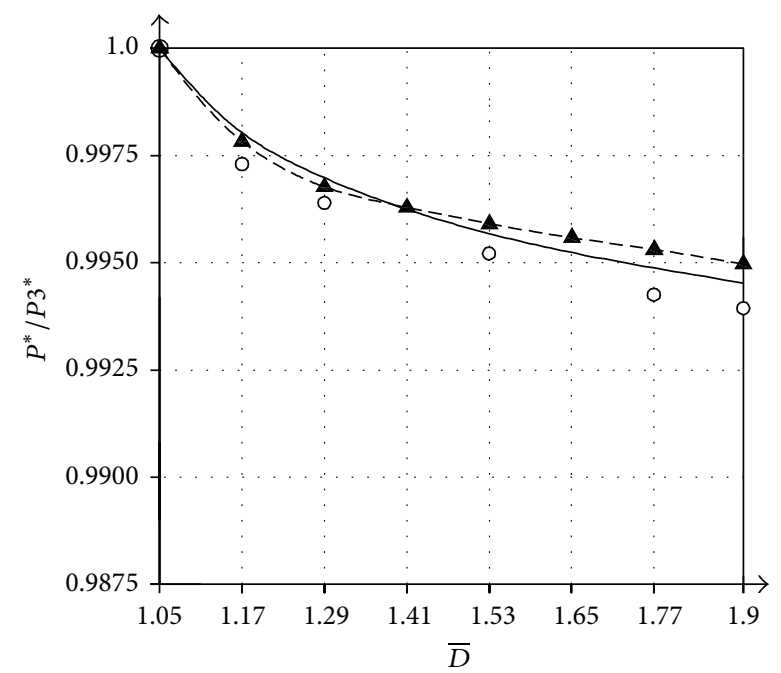

(b) $\alpha_{3}=30.0^{\circ}$

FIGURE 7: Calculated and experimental averaged total pressure: ooo measured; - $\mathbf{\Delta}$ - calculated (Ansys CFX); - - - calculated (the own-designed model).

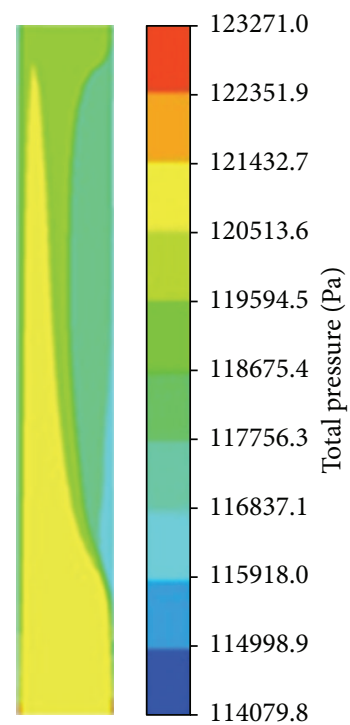

(a) $\alpha_{3}=16.8^{\circ}$

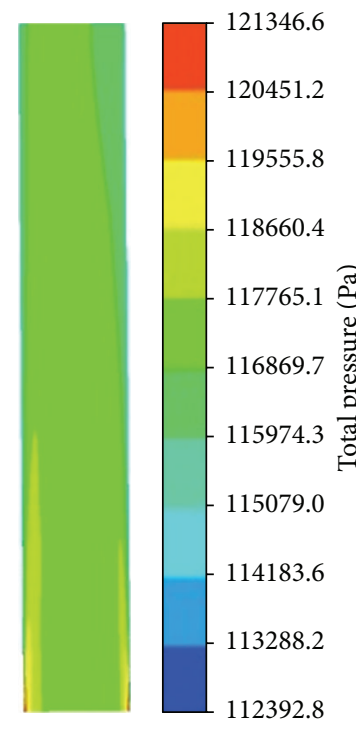

(b) $\alpha_{3}=22.4^{\circ}$

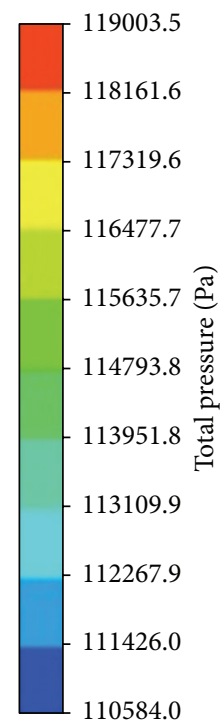

(c) $\alpha_{3}=30.0^{\circ}$

FIgURE 8: Total pressure distributions across the diffuser.

measured ones. Figure 8 shows the distributions of the total pressure at cross-section of the diffuser at different inlet flow angles, identifying that high losses occur in the separation zone.

An increase in measured total pressure losses at $\alpha_{3}=$ $30.0^{\circ}$ is apparently also caused by jet-wake mixing. Figure 9 shows the streamlines in relative frame at different spanwise transversal surfaces at different flow rates which exhibit a jet/wake structure at the impeller outlet. The frozen rotor interface was used for these calculations, as an alternative to the stage interface.

It can be seen that at $\alpha_{3}=30.0^{\circ}$ the flow around the blades is favorable, and the wake area is almost inconspicuous. At $\alpha_{3}=16.8^{\circ}$ on the suction side of the blade near the shroud a zone of recirculation is formed. As a result, an extensive wake flow was detected at the impeller outlet. At $\alpha_{3}=36.2^{\circ}$ a small velocity wake at the shroud-suction side corner of the impeller exit was identified.

Figure 10 shows the total pressure in relative frame distributions near the trailing edge. As the diagram shows at off-design operating conditions, the results depict a strong nonuniform total pressure distribution at the impeller outlet extending downstream to the diffuser and resulting in higher jet-wake mixing losses.

As it was shown, jet-wake mixing strongly affects the total pressure loss, especially at the off-design point. This feature 

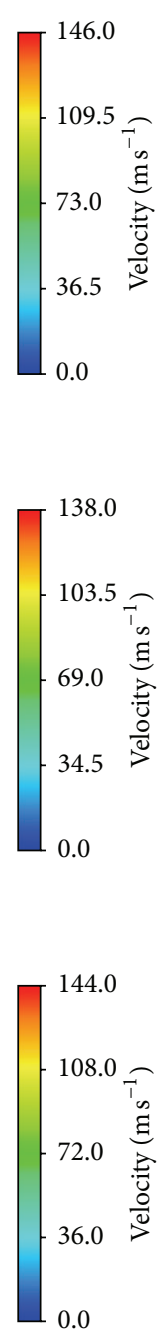

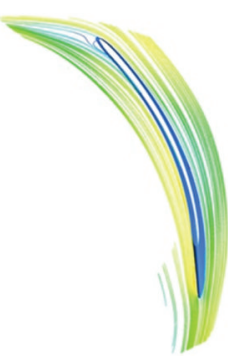

Hub side

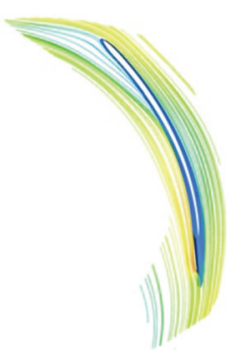

Mid-span

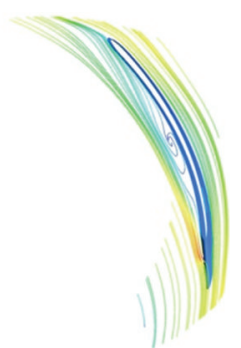

Shroud side

(a) $\alpha_{3}=16.8^{\circ}$
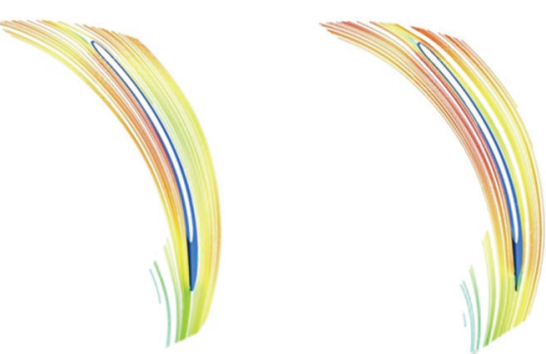

Mid-span

(b) $\alpha_{3}=30.0^{\circ}$

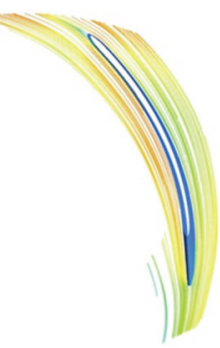

Hub side

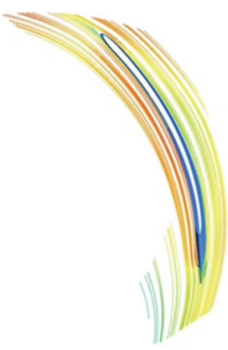

Mid-span

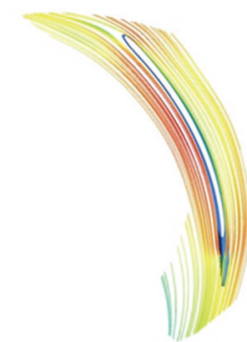

Shroud side

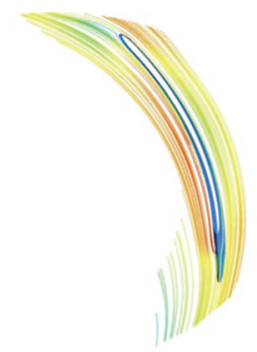

Shroud side

(c) $\alpha_{3}=36.2^{\circ}$

FIGURE 9: Impeller streamline patterns in relative frame.

explains the difference between the measured and calculated total pressure loss coefficients. To predict the true interaction between the impeller and the diffuser the transient simulation has to be used. The main disadvantage of this method is the fact that unsteady simulations are far more computationally expensive.

Summing up the above, it should be noted that at high flow rates the total pressure loss increases because of intensive jet-wake mixing, while at low flow rates the efficiency of the vaneless diffusers decrease mostly because of the flow separation. It was also found that when reverse flow at the diffuser outlet extends close to the entry region the rotating stall is initiated [17]. It results in dramatical loss of compressor performance and instability and even can cause damage of the machine.

Therefore, to decrease total pressure loss coefficient at low flow rates and to improve centrifugal compressor stability boundary layer control techniques should be used. Many researchers have contributed to determine control mechanisms for boundary separation, stall, and surge in centrifugal compressors over the past several years. Some of them $[18,19]$ had shown that an improvement in stable range of the centrifugal compressors could be obtained by injecting air through the shroud and hub surface of the diffuser. Furthermore some researchers $[1,2,20]$ found that decrease in width ratio helps to avoid rotating stall. That is why the purpose of our further investigation is to improve diffuser efficiency and compressor stability using pitched diffusers and injecting air through both the diffuser hub and shroud surfaces.

\section{Conclusions}

Experimental and numerical investigations of the vaneless diffuser were performed. As the results show, at low flow rates the total pressure loss increases because of higher frictional, separation, and jet-wake mixing losses, while at higher flow rates it results from the jet-wake mixing. 

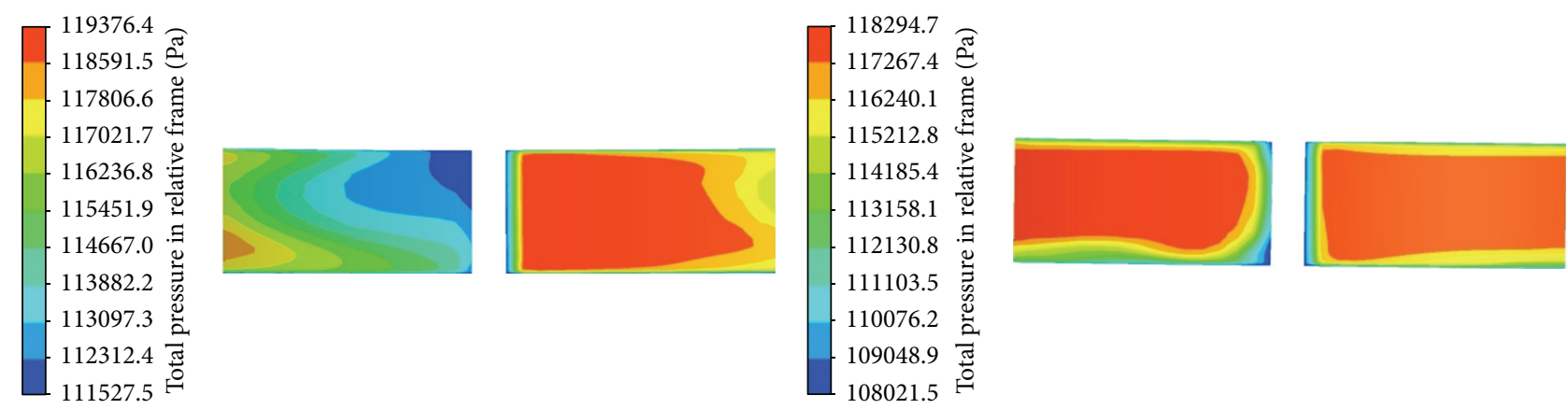

(a) $\alpha_{3}=16.8^{\circ}$

(b) $\alpha_{3}=30.0^{\circ}$
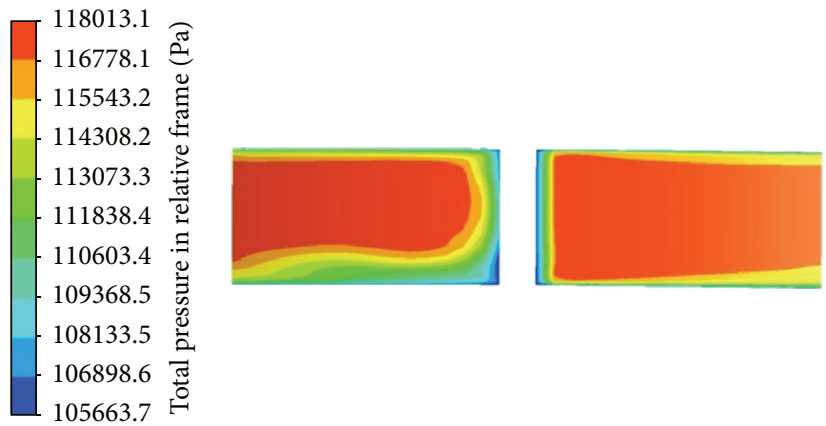

(c) $\alpha_{3}=36.2^{\circ}$

FIGURE 10: Total pressure distributions in relative frame near the trailing edge.

It was shown that the steady-state models allow to get good results, especially near the best efficiency point. However, these models do not account the true interaction between the rotor and stator. In some cases, modeling these aspects is the key to getting accurate solutions. That is why at off-design points the unsteady simulation seems to be more preferable.

The own-designed model showed a significant qualitative and quantitative correspondence between calculated and experimental data at the design point, and the separation point was estimated rather well.

To decrease the total pressure loss at low flow rates and to improve centrifugal compressor stability, boundary layer control techniques, such as injection and pitched diffusers, should be used.

\section{Nomenclature}

$c_{r}, c_{u}, c: \quad$ Radial velocity, circumferential velocity, and absolute velocity, $\mathrm{m} / \mathrm{s}$

$C_{r}, C_{u}, C:$ Averaged radial velocity, averaged circumferential velocity, and averaged absolute velocity, $\mathrm{m} / \mathrm{s}$

D: $\quad$ Diameter, $\mathrm{m}$

$\bar{D}=D / D_{2}:$ Relative diameter

$\tau: \quad$ Tangential stress, $\mathrm{Pa}$

p: $\quad$ Averaged static pressure, $\mathrm{Pa}$

$p^{*}: \quad$ Averaged total pressure, $\mathrm{Pa}$

$\rho: \quad$ Averaged density, $\mathrm{kg} / \mathrm{m}^{3}$

$b: \quad$ Diffuser width, $\mathrm{m}$ $\bar{b}=b / D_{2}: \quad$ Width ratio

$\alpha: \quad$ Averaged flow angle, ${ }^{\circ}$

$C_{p}: \quad$ Static pressure recovery coefficient

$\zeta: \quad$ Total pressure loss coefficient

$\delta: \quad$ Boundary layer thickness, $\mathrm{m}$

$\tau_{0}: \quad$ Tangential stress on the wall, $\mathrm{Pa}$

$v$ : Kinematic viscosity, $\mathrm{m}^{2} / \mathrm{s}$

$z: \quad$ Distance to the wall, $\mathrm{m}$

$\eta=z / \delta: \quad$ Dimensionless coordinate

$c_{*}=\sqrt{\tau_{0} / \rho}:$ Friction velocity, $\mathrm{m} / \mathrm{s}$

$v=c / c_{*}: \quad$ Dimensionless velocity

$\operatorname{Re}_{*}=c_{*} \delta / \nu$ : Specific Reynolds' number

$\delta_{r}^{*}$ : $\quad$ Displacement thickness, $\mathrm{m}$

$\delta_{r}^{* *}: \quad \quad$ Momentum thickness, $\mathrm{m}$

$\Gamma$ : $\quad$ The Buri shape-factor

$\chi=0.4: \quad$ Prandtl number.

Subscripts

2: Impeller outlet

3: Diffuser inlet

4: Diffuser outlet

sub: Viscous sublayer boundary

$m$ : Boundary of the boundary layer.

\section{Conflict of Interests}

The authors declare that there is no conflict of interests regarding the publication of this paper. 


\section{References}

[1] W. Jansen, "Rotating stall in a radial vaneless diffuser," Journal of Basic Engineering, vol. 86, pp. 750-758, 1964.

[2] Y. Senoo and Y. Kinoshita, "Influence of inlet flow conditions and geometries of centrifugal vaneless diffusers on critical flow angle for reverse flow," Journal of Fluid Mechanics, vol. 99, no. 1, pp. 98-103, 1977.

[3] Y. Tsujimoto, Y. Yoshida, and Y. Mori, "Study of vaneless diffuser rotating stall based on two-dimensional inviscid flow analysis," Journal of Fluids Engineering, vol. 118, no. 1, pp. 123-127, 1996.

[4] S. Ljevar, H. C. de Lange, and A. A. van Steenhoven, "Twodimensional rotating stall analysis in a wide vaneless diffuser," International Journal of Rotating Machinery, vol. 2006, Article ID 56420, 11 pages, 2006.

[5] A. S. Nuzhdin, Investigation of vaneless diffusers of centrifugal compressors [Ph.D. thesis], Leningrad Polytechnic University, Leningrad, USSR, 1969 (Russian).

[6] A. N. Abdelhamid, "Effects of vaneless diffuser geometry on flow instability in centrifugal compression systems," Canadian Aeronautics and Space Journal, vol. 29, no. 2, pp. 259-266, 1983.

[7] Y. Kinoshita and Y. Senoo, "Rotating stall induced in vaneless diffusers of very low specific speed centrifugal blowers," Journal of Engineering for Gas Turbines and Power, vol. 107, no. 2, pp. 514-521, 1985.

[8] A. Jaatinen-Varri, P. Roytta, T. Turunen-Saaresti, and A. Gronman, "Experimental study of centrifugal compressor vaneless diffuser width," Journal of Mechanical Science and Technology, vol. 27, no. 4, pp. 1011-1020, 2013.

[9] C. Gao, C. Gu, T. Wang, and Z. Dai, "Numerical analysis of rotating stall characteristics in vaneless diffuser with large width-radius ratio," Frontiers of Energy and Power Engineering in China, vol. 2, no. 4, pp. 457-460, 2008.

[10] S. Khalfallah and A. Ghenaiet, "Impeller-vaneless-diffuserscroll interactions and unsteady flow analysis in a centrifugal compressor," in Proceedings of the 6th International Conference on Compressors and their Systems, pp. 177-193, London, UK, September 2009.

[11] R. A. Izmailov, H. D. Lopulalan, and G. S. Norimarna, "Numerical modelling of unsteady flow phenomena in a centrifugal compressor stage," Compressor Equipment and Pneumatics, vol. 5, pp. 10-15, 2011 (Russian).

[12] H. Tamaki, "Study on flow fields in high specific speed centrifugal compressor with unpinched vaneless diffuser," Journal of Mechanical Science and Technology, vol. 27, no. 6, pp. 16271633, 2013.

[13] M. Kalinkevych and O. Shcherbakov, "Experimental investigation of gas flow in the vaneless diffuser of centrifugal compressor," DonNTU Herald, vol. 22, pp. 88-100, 2011 (Russian).

[14] M. Kalinkevych, O. Shcherbakov, O. Gusak, and V. Ihnatenko, "Investigation of the gas flow in the vaneless diffusers of the centrifugal compressors," in Proceedings of the 7th International Conference on Compressors and their Systems, pp. 5-6, London, UK, September 2011.

[15] A. N. Sherstyuk, The Turbulent Boundary Layer, Energiya, Moscow, Russia, 1974 (Russian).

[16] R. C. Dean and Y. Senoo, "Rotating wakes in vaneless diffusers," Journal of Basic Engineering, vol. 82, pp. 563-570, 1960.

[17] H.-S. Dou and S. Mizuki, "Analysis of the flow in vaneless diffusers with large width-to-radius ratios," Journal of Turbomachinery, vol. 120, no. 1, pp. 193-201, 1998.
[18] Z. S. Spakovszky, H. J. Weigl, J. D. Paduano, C. M. Van Schalkwyk, K. L. Suder, and M. M. Bright, "Rotating stall control in a high-speed stage with inlet distortion: part I-radial distortion," Journal of Turbomachinery, vol. 121, no. 3, pp. 510-516, 1999.

[19] G. J. Skoch, "Experimental investigation of centrifugal compressor stabilization techniques," Journal of Turbomachinery, vol. 125, no. 4, pp. 704-713, 2003.

[20] T. Turunen-Saaresti, A. Reunanen, and J. Larjola, "Computational and experimental study of pinch on the performance of a vaneless diffuser in a centrifugal compressor," Journal of Thermal Science, vol. 15, no. 4, pp. 306-313, 2006. 

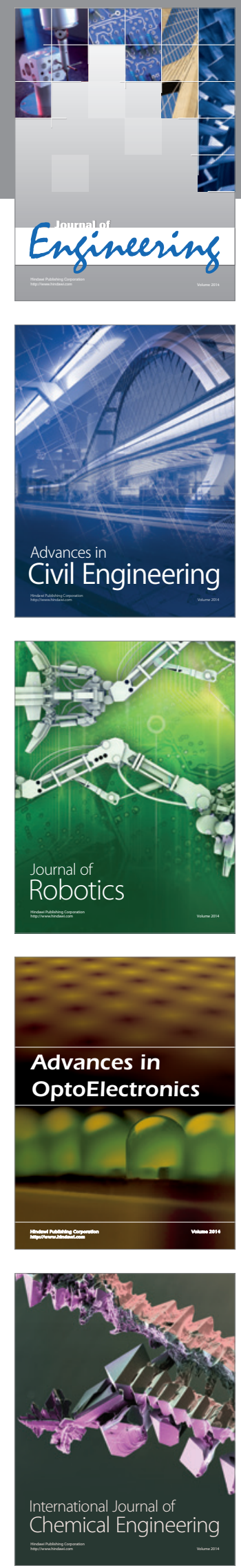

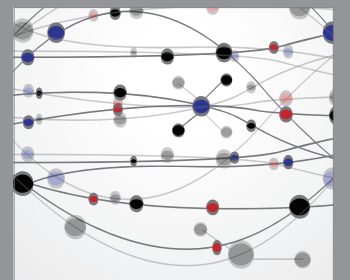

The Scientific World Journal
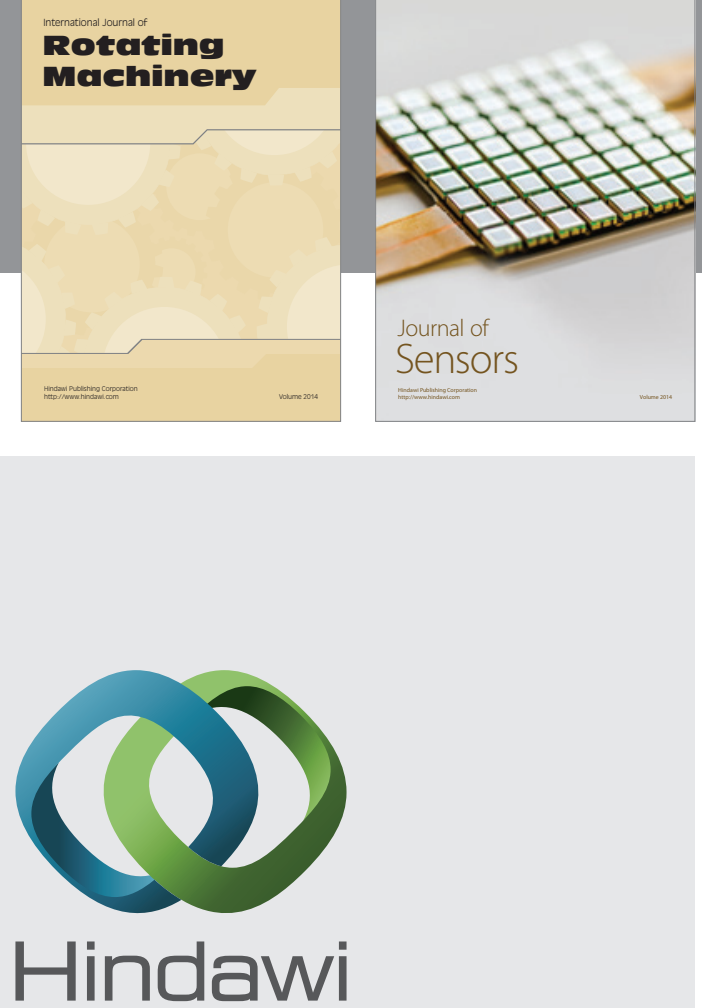

Submit your manuscripts at http://www.hindawi.com
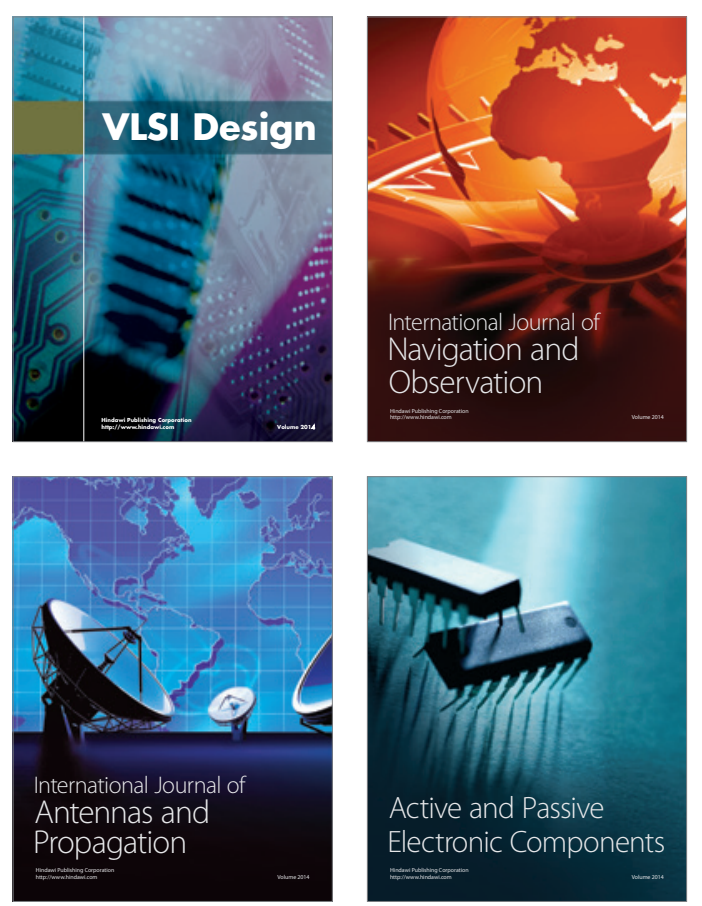
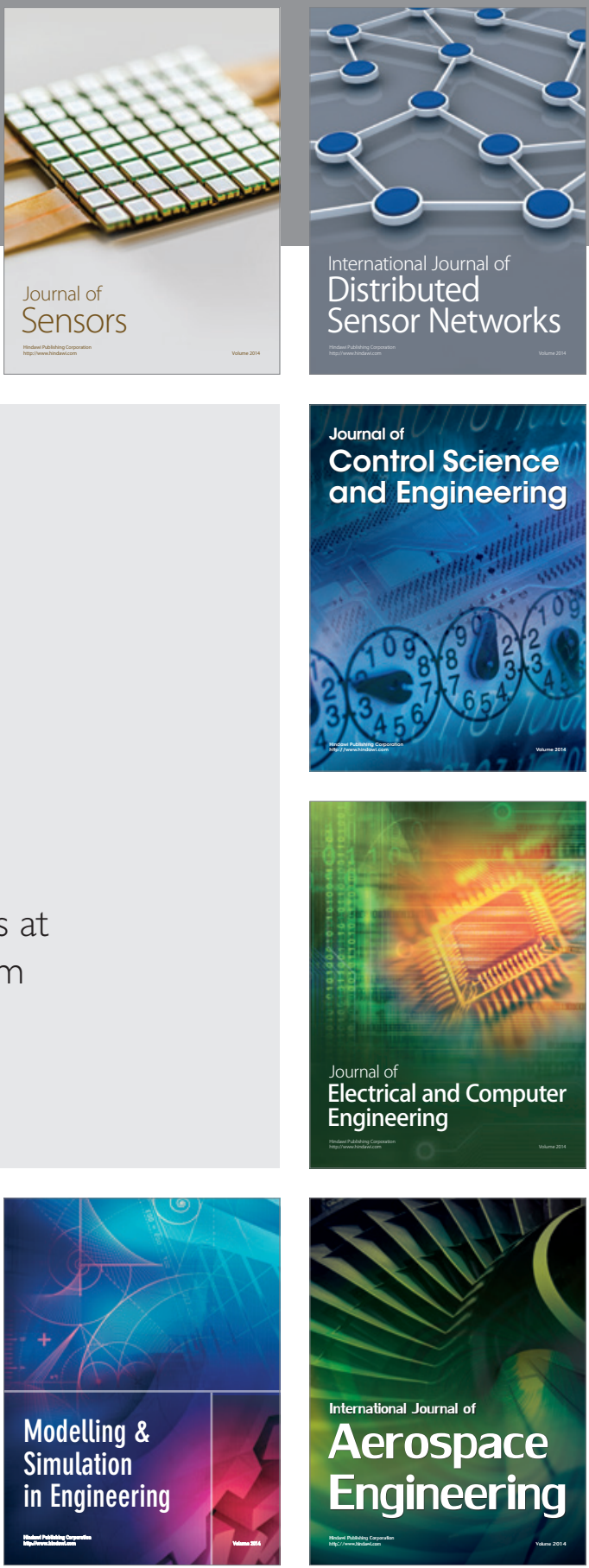

Journal of

Control Science

and Engineering
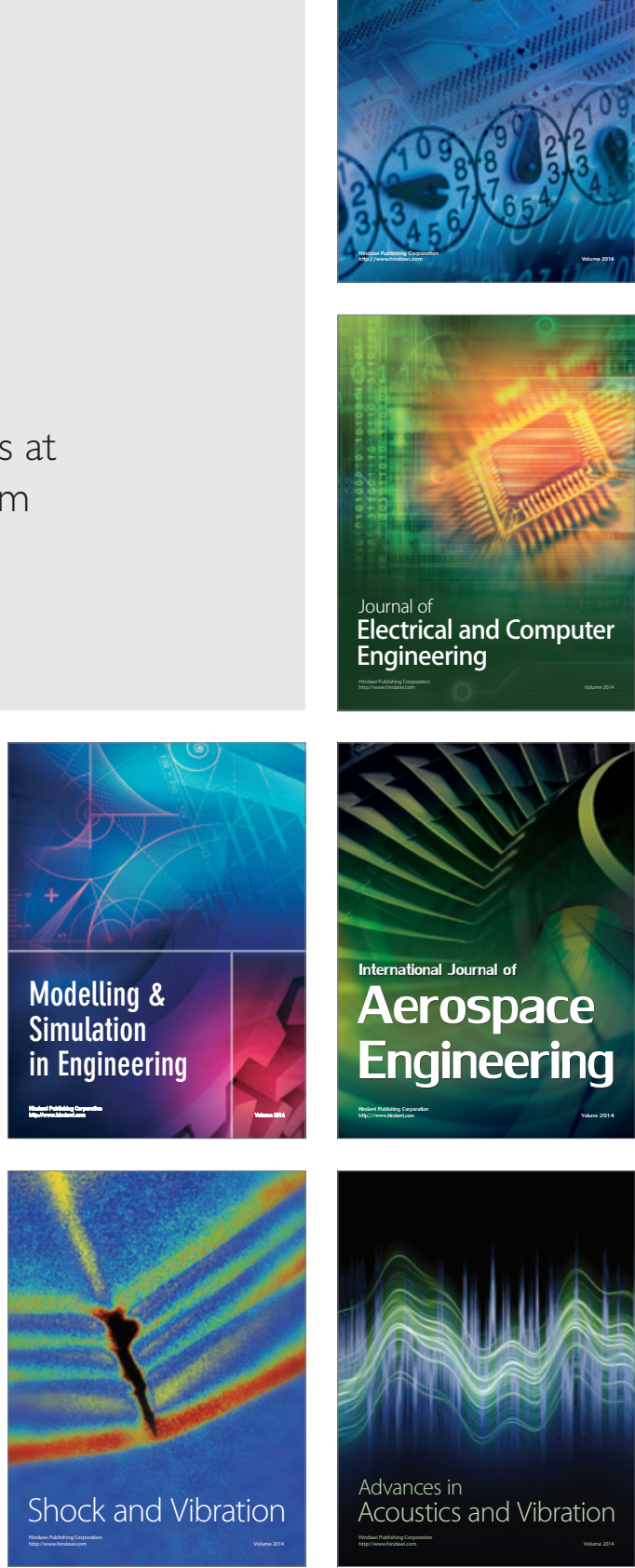